\title{
The electro-oculogram in human retinal detachment
}

\author{
LOUIS A. LOBES \\ From the Department of Ophthalmology, University of Iowa Hospitals and Clinics, Iowa City, Iowa, USA
}

SUMMARY Thirteen patients with retinal detachment and surgical reattachment were studied with preoperative and multiple postoperative electro-oculograms. The light rise was reduced to a mean of 1.17 with detachment but recovered in $77 \%$ to a 1.87 ratio, the equivalent of the control eye. This recovery was complete within 66 days in $80 \%$ of patients who eventually did return to normal. This rapid recovery parallels the rapid return of anatomical, biochemical, and electrophysiological function demonstrated in experimental detachment.

The alterations to the electro-oculogram (EOG) caused by retinal detachment were first described by Heck and Papst (1957), and Arden (1962). The work was confirmed by Foulds and Ikeda (1966) in the rabbit. Blach and Behrman (1967), and Schmidt (1970) then reported conflicting results on the timing and actual return of the EOG after retinal reattachment. Machemer $(1961 \mathrm{a}, \mathrm{b})$, and Kroll and Machemer (1971) in an experimental model demonstrated a rapid and thorough anatomical recovery of rod and cone outer segments within 4 weeks of reattachment. Hamasaki et al. (1969) showed that the electrophysiological recovery was also rapid and complete.

This experimental model would suggest that the EOG should also fully return to normal after retinal reattachment and that it should do so rapidly. Yet the clinical studies cited disagree on the actual return of the EOG to a normal value and suggest at least a year's recovery before the return to normal EOG values could be attained.

The present study was therefore organised to investigate further the electro-oculographic characteristics of human retinal detachment and its surgical reattachment.

\section{Materials and methods}

Thirteen patients with primary retinal detachment, with the macula already involved, and unassociated with choroidal detachment, were selected from the retina clinic at the University of Iowa Hospitals and Clinics. Clinical EOG measurements were made after dilation with $1 \%$ cyclopentolate and $10 \%$

Address for reprints: Dr Louis A. Lobes, Department of Ophthalmology, Eye and Ear Hospital of Pittsburgh, 230 Lothrop Street, Pittsburgh, Pennsylvania 15213, USA phenylephrine. The following protocol for EOG measurements was employed (Kolder and Hochesand, 1973). The patient was placed in a dim room. Beckman skin electrodes at the medial and lateral canthus and forehead were connected to a direct-current amplifier. The input to the amplifier was clamped for 8 seconds out of every 10 seconds. During the 2 seconds when the input was open the potential was measured while the patient followed 2 red fixation lights subtending an angle of $30^{\circ}$ and placed at a distance of $1 \mathrm{~m}$. Those potentials were amplified and monitored on an oscilloscope and at the same time fed into a Helcoscriptor paper recorder running at a paper speed of $2 \mathrm{~mm} / \mathrm{min}$.

The light source for the EOG light consisted of four $24-\mathrm{V}$ incandescent bulbs of $30 \mathrm{~W}$ mounted behind an opalescent glass plate subtending an area of approximately $1400 \mathrm{~cm}^{2}$ at a distance of $1 \mathrm{~m}$ from the patient.

Once the polarisation current between electrodes and skin stabilised, the EOG lights were turned off and a dark trough oscillation was induced. After 12.5 minutes the light source was turned on and the light rise observed.

EOGs were recorded preoperatively in all 13 patients who had successful retinal reattachment. Postoperative recordings were performed at varying intervals.

In the EOG laboratory at the University of Iowa the mean for the light peak/dark trough ratio in normal individuals is $2 \cdot 00$, with a standard deviation of 0.3 . Therefore ratios between 1.4 and 2.6 include $95 \%$ of the normal population. EOG ratios below 1.4 are considered abnormal.

The extent of retinal detachment was determined by the number of clock hours shown to be detached on the preoperative retinal drawing. 


\section{Results}

The study group of 13 patients comprised 6 females and 7 males. The mean age was $57 \cdot 2$, with a range from 9 years to 77 years. All presented with primary retinal detachments. Eight patients were emmetropic, 2 had highly myopic refractive errors ( $>-6$ dioptres), and 3 were aphakic.

Table 1 presents the light peak/dark trough ratio among the 13 patients, comparing their normal eye with the eye with retinal detachment. The mean ratio in the detached eye was $1 \cdot 17$, with a standard deviation of $\pm 0 \cdot 157$, while the mean ratio in the normal eye was $1 \cdot 97$. Student's $t$ difference test shows this difference to be significant at $P<0.001$. The mean of 1.17 was also 2.8 standard deviations from the normal.

After successful retinal reattachment the EOG ratio of the study eyes was compared with that of the controls (Table 2). The time interval between the preoperative and postoperative measurements was also included. In 9 patients the EOG returned to a mean of 1.87 , which is a normal recording in this laboratory. Three patients had no return as of their last recording of 2 to 3 months. The overall mean of the reattached eyes was 1.67. Fig. 1 shows the changes in individual eyes.

The early return of the light rise was studied more closely in 3 patients (Table 3 ). The earliest return to an EOG ratio equivalent to the control eyes was at 30 days (Table 2 ). The return seemed to be a gradual increase in the 3 patients studied. Only patient No. 8 had a decrease in his ratio from a baseline preoperative of 1.30 to 1.004 days postoperative.

One patient had a detachment of 1 quadrant ( 3 clock hours). His EOG ratio was $1 \cdot 42$. Six patients had 2 quadrants ( 6 clock hours) detached, and their mean ratio was $1 \cdot 20$. Two patients had 3 quadrants detached with a mean EOG of $1 \cdot 10$, and 4 patients with total detachments had a mean EOG ratio of 1.07. There was no correlation between the initial or final visual acuity and the initial or final EOG.

Estimation of the duration of the retinal detachment was obtained from 10 patients (Table 4). The mean EOG ratio for those detached between 1 week and 1 month was $1 \cdot 20$.

\section{Discussion}

The findings in this study suggest that the light peak of the EOG is significantly reduced by retinal detachment. The convenience of the 'other' eye as control allowed firm statistical confirmation of this observation.

Blach and Behrman (1967) observed that, with $35 \%$ or more of the retina detached, the light rise was minimal in 3 patients and totally absent in 12 patients. They also observed for the first time a gradual rise in the EOG beginning 4 months after surgical reattachment. The EOG did not approach the patient's 'normal' eye until 1 year after operation.

Table 1 EOG in patients with retinal detachment

\begin{tabular}{lll}
\hline Patient & Detached eye & Control eye \\
\hline 1 & 1.00 & 1.90 \\
2 & 1.31 & 2.00 \\
3 & 1.06 & 1.55 \\
4 & 1.00 & 2.00 \\
5 & 1.42 & 2.00 \\
6 & 1.20 & 2.22 \\
7 & 1.00 & 2.16 \\
8 & 1.30 & 2.00 \\
9 & 1.00 & 2.07 \\
10 & 1.20 & 2.25 \\
11 & 1.20 & 1.55 \\
12 & 1.50 & 2.00 \\
13 & 1.00 & 2.00 \\
\hline Mean & 1.17 & 1.97 \\
Standard deviation & \pm 0.157 \\
\hline & & \\
\hline
\end{tabular}

Table 2 EOG in patients after successful retinal reattachment

\begin{tabular}{lccc}
\hline Patient & Detached eye & Control eye & Time interval \\
\hline 1 & 1.00 & 2.00 & 3 months \\
2 & 1.50 & 2.00 & 8 days \\
3 & 1.00 & 1.50 & 3 months \\
4 & 1.00 & 2.00 & 2 months \\
5 & 1.83 & 2.00 & 1.3 months \\
6 & 2.00 & 2.00 & 2.5 months \\
7 & 2.00 & 2.00 & 2.8 months \\
8 & 2.00 & 2.00 & 2.5 months \\
9 & 2.00 & 2.50 & 3 months \\
10 & 2.00 & 2.00 & 1 month \\
11 & 2.00 & 2.00 & 4 months \\
12 & 1.90 & 2.00 & 7 months \\
13 & 1.50 & 2.00 & 7 months \\
\hline Mean & 1.67 & 2.00 & \\
\hline
\end{tabular}


Table 3 Time interval for return of the EOG to normal in eyes with retinal reattachment

\begin{tabular}{lll}
\hline Patient & Time & EOG \\
\hline 8 & Preoperative & 1.30 \\
& 4 Days postoperative & 1.00 \\
& 16 Days postoperative & 1.28 \\
& 72 Days postoperative & 2.00 \\
& & 1.20 \\
11 & Preoperative & 1.33 \\
& 7 Days & 1.33 \\
& 15 Days & 2.00 \\
& 144 Days & 1.00 \\
7 & Preoperative & 1.38 \\
& 12 Days & 2.00 \\
\hline
\end{tabular}

Table 4 Relationship of duration of retinal detachment to the EOG ratio

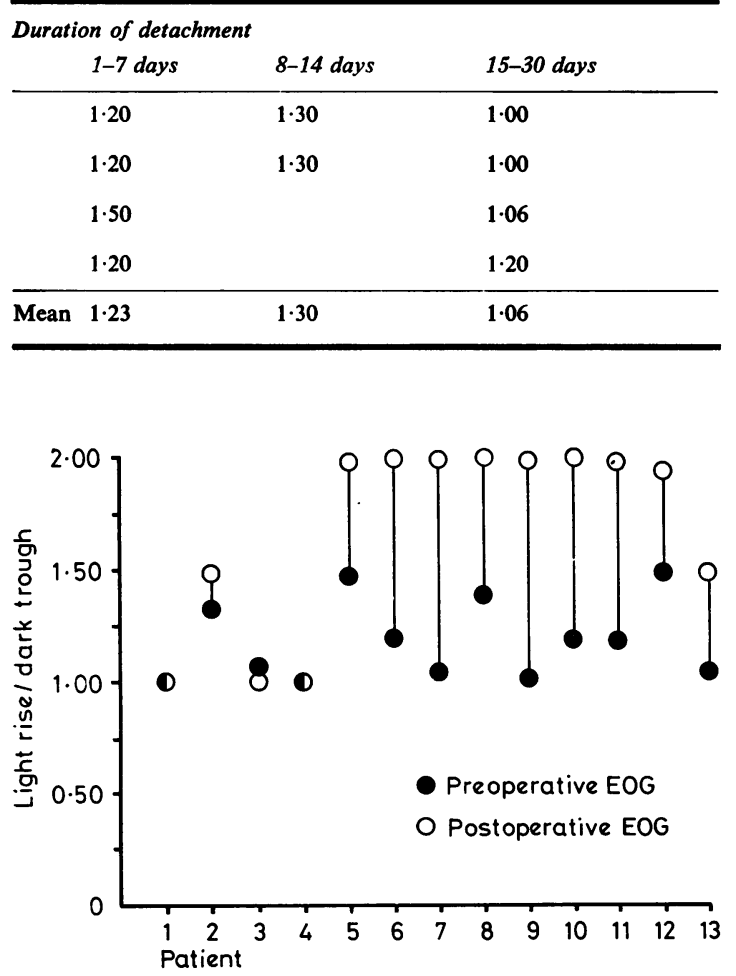

Figure The comparison of preoperative to postoperative EOGs in 13 eyes with detachment and successful repair
In Schmidt's (1970) series, however, the mean EOG for the detached eyes remained pathological, although it did increase minimally after successful reattachment. The return of the EOG was delayed as long as $\mathbf{8 0 0}$ days in some patients. She noted in her groups with $25 \%$ and total detachments that the EOG actually decreased from detachment to successful reattachment.

The EOG in this study underwent a statistically significant recovery with surgical reattachment of the retina. Of our patients $77 \%$ had the EOG return to a mean of 1.87 , statistically indistinguishable from the 'control' eyes and well within the $95 \%$ confidence limit $(1.4$ to 2.6$)$ for a normal EOG. In 6 patients the return occurred by $2 \cdot 2$ months. The overall mean EOG for all our reattached patients was also normal for this laboratory $(1 \cdot 67)$. The return appeared to be a gradual process in 3 patients closely observed.

Machemer (1968a, b), and Kroll and Machemer (1971) documented in their experimental retinal detachment model that the physiological recovery process began actively within 3 hours of reattachment. The rod and cone outer and inner segments were found in almost perfectly parallel array within 4 weeks of reattachment, and by 12 weeks there was no histological evidence of a retinal detachment in areas not included in the scleral buckle (Machemer, 1968c). Hamasaki et al. (1969) showed that this anatomical return to normal was also paralleled by an electrophysiological return to 'normal' when he described the linear, time-related return of the originally extinguished electroretinogram after retinal reattachment. The greatest improvement occurred in the first 14 days after surgery.

The mean recovery time of our patients with returned EOG ratio was 90 days, while $80 \%$ of these had returned within 66 days. The previous studies (Blach and Behrman, 1967; Schmidt, 1970) did not note such a rapid return nor in such a high percentage of patients, but our present data seem more in keeping with the well-documented experimental studies that would predict a return within a few months rather than a year or more.

While several of Schmidt's (1970) patients showed an actual decrease in EOG potential with reattachment of the retina, only patient No. 8 showed such a decrease, and this was rapidly reversed to a fully recovered 2.00 ratio by 2.5 months.

We were unable to explain satisfactorily why the EOG ratio did not return in some patients after retinal reattachment, but age, sex, size, or duration of detachment, and refractive error were not significant contributing factors. Perhaps as in previously published cases there will need to be a year or more for full physiological recovery. 
Because there were so few patients in each group and because their mean EOG ratios were so similar, we were unable to assess the influence that the size of the detachment has on the EOG. We did confirm that less than $50 \%$ detachment can reduce the EOG to 1.00 (patients Nos. 9 and 13), but this was not a steadfast rule as Blach and Behrman (1967) implied. Patient No. 12 with a total detachment had an EOG of $1 \cdot 31$. His retina had been detached for 8 to 14 days, and patient No. 12 had 5 clock hours retinal detachment for 10 days, and his EOG was 1.50. Therefore individual variations exert an important influence on these data.

The duration of the retinal detachment did not seem to be an important influence on the EOG ratio. Four patients, whose retina had been detached for less than 1 week, had a mean EOG of $1 \cdot 23$. The small number precluded statistical inference here.

This study confirms that the EOG is truly reduced by retinal detachment and that this diminished EOG ratio can return to the equivalent of the control eye. This is a gradual recovery, but one that begins in the early postoperative period and in many patients is completed by 66 days. These findings are consistent with the anatomical, physiological, and electroretinographic (ERG) recovery that Machemer (1968a, b, c), Kroll and Machemer (1971), and Hamasaki (1969) have shown to occur with experimental detachment.

The EOG would therefore seem to be an adjunct for confirming the diagnosis of retinal detachment and reattachment among patients with opaque media when the ERG and quantitative A-scan are equivocal or unavailable.

\section{References}

Arden, G. B., and Kelsey, J. H. (1962). Some observations on the relationship between the standing potential of the human eye and the bleaching regeneration of the visual purple. Journal of Physiology, 161, 205-226.

Blach, R. K., and Behrman, J. (1967). The electrical activity of the eye in retinal detachment. Transactions of the Ophthalmological Societies of the United Kingdom, 89, 263-266.

Foulds, W. S., and Ikeda, H. (1966). The effects of detachment of the retina on the induced and resting ocular potentials in the rabbit. Investigative Ophthalmology, 5, 93-108.

Hamasaki, D. I., Machemer, R., and Norton, E. W. D. (1969). Experimental retinal detachment in the owl monkey, VI. The ERG of the detached and reattached retina. Albrecht von Graefes Archiv für klinische und experimentelle Ophthalmologie, 177, 212-217.

Heck, J., and Papst, W. (1957). Uber den Ursprung des corneoretinalen Ruheopotentials. Bibliotheca Ophthalmologica, 48, 96-107.

Kolder, H. F., and Hochesand, D. (1973). Emperical model of electro-oculogram. Documenta Ophthalmologica, 34, 229-241.

Kroll, A. J., and Machemer, R. (1971). Experimental retinal detachment in the owl monkey, VIII. Photoreceptor protein renewal in early retinal reattachment. American Journal of Ophthalmology, 72, 356-366.

Machemer, R. (1968a). Experimental retinal detachment in the owl monkey, II. Histology of the retinal and pigment epithelium. American Journal of Ophthalmology, 66, 410423.

Machemer, R. (1968b). Experimental retinal detachment in the owl monkey. III. Electron microscopy of retina and pigment epithelium. American Journal of Ophthalmology, 66, 410-423.

Machemer, R. (1968c). Experimental retinal detachment in the owl monkey, IV. The reattached retina. American Journal of Ophthalmology, 66, 1075-1091.

Schmidt, B. (1970). Elektro-oculographische Untersuchungen bei ablatio retinae. Albrecht von Graefes Archiv für klinische und experimentelle Ophthalmologie, 180, 20-30. 